\title{
Minimum polyhedron with $n$ vertices
}

\author{
Shigeki AkiYama
}

(Received October 21, 2018)

(Revised January 28, 2021)

\begin{abstract}
We study a polyhedron with $n$ vertices of fixed volume having the minimum surface area. Completing the proof of Fejes Tóth, we show that all faces of a minimum polyhedron are triangles, and further prove that a minimum polyhedron does not allow deformation of a single vertex. We also present possible minimum shapes for $n \leq 12$. Some of them are quite unexpected, in particular $n=8$.
\end{abstract}

\section{Introduction}

Let $X$ be a closed set in $\mathbb{R}^{d}$. Denote by $V_{d}(X)$ the $d$-dimensional Lebesgue measure of $X$ and by $A_{d}(X)$ the surface area of $X$, i.e., $d-1$ dimensional Lebesgue measure of $\partial(X)$. For a non-empty set $A$ in $\mathbb{R}^{d}$, we denote by $\Delta(A)$ the convex hull of $A$. A convex body in $\mathbb{R}^{d}$ is a compact convex set with a non-empty interior. For a convex body $X$, we recall the isoperimetric inequality:

$$
\frac{A_{d}(X)}{V_{d}(X)^{(d-1) / d}} \geq \frac{A_{d}\left(B^{d}\right)}{V_{d}\left(B^{d}\right)^{(d-1) / d}},
$$

where $B^{d}$ is the $d$-dimensional unit ball (c.f. [12]). The equality is attained only when $X$ is a $d$-dimensional ball. Note that if $X$ is a planar convex set, then in the plain language, $V_{2}(X)$ is the area and $A_{2}(X)$ is the perimeter of $X$.

Let $d=3$ and $n \geq 4$. We are interested in minimizing $A_{3}(X) / V_{3}(X)^{2 / 3}$ among all polyhedra $X$ with $n$ vertices. Clearly we may assume that $X$ is convex. Denote by $\Delta_{n}=\Delta\left(p_{1}, \ldots, p_{n}\right)$ the convex hull of $n$ points $p_{1}, p_{2}, \ldots$, $p_{n} \in \mathbb{R}^{3}$. We say $\Delta_{n}$ is non-degenerate if $V_{3}\left(\Delta_{n}\right)>0$. Therefore our problem is to minimize $A_{3}\left(\Delta_{n}\right) / V_{3}\left(\Delta_{n}\right)^{2 / 3}$ among all non-degenerate convex hull $\Delta_{n}$ 's of $n$ points in $\mathbb{R}^{3}$. We are of course interested in the shape $\Delta_{n}$ which attains its minimum as well. Since $A_{3}\left(\Delta_{n}\right) / V_{3}\left(\Delta_{n}\right)^{2 / 3}$ is invariant under similitudes, our problem is equivalent to find the minimum $A_{3}\left(\Delta_{n}\right)$ under $V_{3}\left(\Delta_{n}\right)=1$. Thus our problem is a discrete variant of the isoperimetric inequality (1), i.e., a

The author is supported by JSPS Grant-in-aids 17K05159, 17H02849, BBD30028.

2010 Mathematics Subject Classification. Primary 52B60, 52B55. 
discrete 'minimum surface'. For a similar minimization problem with a given number of faces, we find several references. Lindelöf [10] and Minkowski [11] proved in different intriguing ways that the minimum polyhedron must be circumscribed about a sphere, and Fejes Tóth [6] proved that the minimum is attained when the number of faces are 4,6 and 12 by the regular tetrahedron, cube and dodecahedron, respectively. Note that minimization with a given number of vertices is a totally different and more difficult problem; e.g., the cube is not a solution for $n=8$ (see Theorems 1 and 3).

Fejes Tóth claimed in [5] and [7, Chapter 5, §7] that every face of the minimum $n$-hedron must be a triangle. However his proof contains a gap due to the fact that the corresponding equi-area piecewise differentiable surface has singular points (see the description around Example 1 for details). We shall classify such singularities (Lemma 3) and complete the proof along his idea in Theorem 1. All the same, digesting his idea, we can further prove that the minimum $n$-hedron does not allow a deformation of a single vertex, by showing that the equi-area body is strictly convex. Finally we give a list of possible shapes of minimum $n$-hedron for $n \leq 12$ by extensive random numerical search together with heavy algebraic computation using Gröbner basis. Conjectural shapes for $n=8$ and $n=11$ may be beyond our imagination.

Added in revision: One of the referees of this paper pointed out that Böröczky and Böröczky Jr [2] gave a proof of Theorem 1 and its generalization. Their proof also rely on the same idea of Fejes Tóth but went in a different way.

\section{Every face is a triangle}

In this section, we prepare basic properties of this minimization problem in Propositions 1 and 2. Then we point out a gap in the proof of Fejes Tóth $[5,7]$ which asserts that every face of the minimum $n$-hedron is triangular. Then we complete the proof after the classification of singular points of the equi-area surface.

Lemma 1. Let $Y$ be a planar polygon in $\mathbb{R}^{3}$ and $g: \mathbb{R}^{3} \rightarrow \mathbb{R}^{2}$ be an orthogonal projection to some plane (e.g., the one along z-axis to xy-plane). Then we have $V_{2}(g(Y)) \leq V_{2}(Y)$ and $A_{2}(g(Y)) \leq A_{2}(Y)$.

Proof. This is clear from the property $\|g(x)-g(y)\| \leq\|x-y\|$ for any $x, y$.

Proposition 1. For a fixed integer $n \geq 4$, the minimum of $A_{3}\left(\Delta_{n}\right) /$ $V_{3}\left(\Delta_{n}\right)^{2 / 3}$ exists where $\Delta_{n}$ varies among non-degenerate convex hulls of $n$ points in $\mathbb{R}^{3}$. 
Proof. Let $R$ be the diameter of $\Delta\left(p_{1}, \ldots, p_{n}\right)$ attained by $\left\|p_{1}-p_{2}\right\|=$ $R$. Let $S$ be the plane passing through $p_{1}$ which is orthogonal to the segment $\left[p_{1}, p_{2}\right]$ and $g$ be the orthogonal projection to $S$. Then $g\left(\Delta_{n}\right)$ is a convex polygon in $S$ with vertices $q_{1}, \ldots, q_{\ell}$ with $\ell \leq n-1$, arranged in the clockwise order with respect to the centroid of $g\left(\Delta_{n}\right)$. Choose $q_{1}^{\prime}, \ldots, q_{\ell}^{\prime}$ in $\Delta_{n}$ such that $g\left(q_{i}^{\prime}\right)=q_{i}$ for $i=1, \ldots, \ell$. If the segment $\left[p_{1}, p_{2}\right]$ lies within $\partial\left(\Delta\left(p_{1}, \ldots\right.\right.$, $\left.p_{n}\right)$ ), we choose $q_{1}^{\prime}=\left(p_{1}+p_{2}\right) / 2$. Since $\Delta_{n}$ is contained in $g\left(\Delta_{n}\right) \times[0, R]$, we have

$$
V_{3}\left(\Delta_{n}\right) \leq V_{2}\left(g\left(\Delta_{n}\right)\right) R
$$

We claim that

$$
A_{3}\left(\Delta_{n}\right) \geq \frac{1}{2} A_{2}\left(g\left(\Delta_{n}\right)\right) R
$$

Since $X \supset Y$ implies $A_{3}(X) \geq A_{3}(Y)$ for convex bodies $X, Y$, considering the surface area of the convex hull $Y=\Delta\left(p_{1}, p_{2}, q_{1}^{\prime}, q_{2}^{\prime}, \ldots, q_{\ell}^{\prime}\right)$, it is enough to prove that

$$
V_{2}\left(p_{1}, q_{i}^{\prime}, q_{i+1}^{\prime}\right)+V_{2}\left(p_{2}, q_{i}^{\prime}, q_{i+1}^{\prime}\right) \geq \frac{1}{2}\left\|q_{i}-q_{i+1}\right\| R
$$

where $V_{2}(x, y, z):=V_{2}(\Delta(x, y, z))$, the area of the triangle of vertices $x, y, z$. The index $i$ of $q_{i}$ is considered modulo $\ell$. Take a plane $P$ containing $p_{1}$ and $p_{2}$ parallel to the segment $\left[q_{i}^{\prime}, q_{i+1}^{\prime}\right]$ and use the orthogonal projection $g_{2}$ to $P$. Noting that the directions of the two projections $g$ and $g_{2}$ are orthogonal, we have $g\left(q_{i}^{\prime}\right)-g\left(q_{i+1}^{\prime}\right)=g\left(g_{2}\left(q_{i}^{\prime}\right)\right)-g\left(g_{2}\left(q_{i+1}^{\prime}\right)\right)$. By Lemma 1, we see

$$
\begin{aligned}
& V_{2}\left(p_{1}, q_{i}^{\prime}, q_{i+1}^{\prime}\right)+V_{2}\left(p_{2}, q_{i}^{\prime}, q_{i+1}^{\prime}\right) \\
& \quad \geq V_{2}\left(p_{1}, g_{2}\left(q_{i}^{\prime}\right), g_{2}\left(q_{i+1}^{\prime}\right)\right)+V_{2}\left(p_{2}, g_{2}\left(q_{i}^{\prime}\right), g_{2}\left(q_{i+1}^{\prime}\right)\right),
\end{aligned}
$$

which is not less ${ }^{1}$ than $\left\|g\left(q_{i}^{\prime}\right)-g\left(q_{i+1}^{\prime}\right)\right\| R / 2$. This shows the claim.

Using (2) and the isoperimetric inequality (1) for $d=2$, that is, $A_{2}\left(g\left(\Delta_{n}\right)\right)^{2} \geq 4 \pi V_{2}\left(g\left(\Delta_{n}\right)\right)$, we see $A_{2}\left(g\left(\Delta_{n}\right)\right) \geq 2 \sqrt{\frac{\pi V_{3}\left(\Delta_{n}\right)}{R}}$. Let us fix $V_{3}\left(\Delta_{n}\right)=$ 1. Then we have $A_{3}\left(\Delta_{n}\right) \geq \sqrt{\pi R}$ from (3). This shows that $A_{3}\left(\Delta_{n}\right) \rightarrow \infty$ as $R \rightarrow \infty$ under the assumption $V_{3}\left(\Delta_{n}\right)=1$. Since we are interested in minimizing $A_{3}\left(\Delta_{n}\right) / V_{3}\left(\Delta_{n}\right)^{2 / 3}$, we may assume that $R$ is bounded by some constant $K$. This shows that parameters $p_{1}, \ldots, p_{n}$ are in a closed ball of radius $K$ with the prescribed property $V_{3}\left(\Delta_{n}\right)=1$. Therefore the set of parameters are in a compact set in $\mathbb{R}^{3}$ and we find the minimum of $A_{3}\left(\Delta_{n}\right)$ as desired.

\footnotetext{
${ }^{1}$ This holds even when $\left[g_{2}\left(q_{i}^{\prime}\right), p_{1}\right]$ and $\left[g_{2}\left(q_{i+1}^{\prime}\right), p_{2}\right]$ intersect.
} 
Therefore we define $\alpha_{n}=\min _{\Delta_{n}} A_{3}\left(\Delta_{n}\right) / V_{3}\left(\Delta_{n}\right)^{2 / 3}$ where $\Delta_{n}$ runs over all non-degenerate convex hulls of $n$ points. A minimum $n$-hedron is the shape $\Delta_{n}$ which attains $\alpha_{n}$. It may not be unique but we expect that it is unique up to similitudes in $\mathbb{R}^{3}$.

Proposition 2. We have $\alpha_{n}>\alpha_{n+1}$ for $n \geq 4$ and $\lim _{n \rightarrow \infty} \alpha_{n}=(36 \pi)^{1 / 3} \approx$ 4.83598 .

Proof. Choose $\Delta_{n}$ which attains $\alpha_{n}$ and its face $T \subset \partial\left(\Delta_{n}\right)$. We take a point $p_{n+1}$ on a outward normal emanating from an inner point $p$ of $T$ whose distance from $T$ is $\varepsilon>0$, which is small enough that $\Delta_{n+1}$ is the union of $\Delta_{n}$ and the pyramid of base $T$ and the apex $p_{n+1}$. Denote by $e_{i}$ the edge of $T$ and $r_{i}$ be the height of $p$ from the edge $e_{i}$ for $i=1,2, \ldots, t$. Note that $r_{i}>0$. Then we see

$$
V_{3}\left(\Delta_{n+1}\right)=V_{3}\left(\Delta_{n}\right)+\frac{1}{3} \varepsilon V_{2}(T)
$$

and

$$
A_{3}\left(\Delta_{n+1}\right)=A_{3}\left(\Delta_{n}\right)-V_{2}(T)+\frac{1}{2} \sum_{i=1}^{t} e_{i} \sqrt{\varepsilon^{2}+r_{i}^{2}} .
$$

Using $V_{2}(T)=\frac{1}{2} \sum_{i=1}^{t} e_{i} r_{i}$, we have

$$
\begin{aligned}
\frac{A_{3}\left(\Delta_{n+1}\right)}{V_{3}\left(\Delta_{n+1}\right)^{2 / 3}} & =\frac{A_{3}\left(\Delta_{n}\right)}{V_{3}\left(\Delta_{n}\right)^{2 / 3}} \frac{1+\frac{1}{2 A_{3}\left(\Delta_{n}\right)} \sum_{i=1}^{t} e_{i} r_{i}\left(\sqrt{1+\left(\frac{\varepsilon}{r_{i}}\right)^{2}}-1\right)}{\left(1+\frac{\varepsilon V_{2}(T)}{3 V_{3}\left(\Delta_{n}\right)}\right)^{2 / 3}} \\
& =\frac{A_{3}\left(\Delta_{n}\right)}{V_{3}\left(\Delta_{n}\right)^{2 / 3}} \frac{1+C_{1} \varepsilon^{2}+O\left(\varepsilon^{3}\right)}{1+C_{2} \varepsilon+O\left(\varepsilon^{2}\right)}
\end{aligned}
$$

with $C_{1}=\frac{1}{4 A_{3}\left(\Delta_{n}\right)} \sum_{i=1}^{t} \frac{e_{i}}{r_{i}}$ and $C_{2}=\frac{2 V_{2}(T)}{9 V_{3}\left(\Delta_{n}\right)}$. Taking small $\varepsilon>0$, we have

$$
\alpha_{n}=\frac{A_{3}\left(\Delta_{n}\right)}{V_{3}\left(\Delta_{n}\right)^{2 / 3}}>\frac{A_{3}\left(\Delta_{n+1}\right)}{V_{3}\left(\Delta_{n+1}\right)^{2 / 3}} \geq \alpha_{n+1} .
$$

By the isoperimetric inequality (1) for $d=3$, we have

$$
A_{3}\left(\Delta_{n}\right) / V_{3}\left(\Delta_{n}\right)^{2 / 3} \geq(36 \pi)^{1 / 3}
$$

and the minimum is sufficiently approximated by points on the sphere, provided $n$ is large.

THEOREM 1. Every face of a minimum n-hedron is a triangle. 
The statement is intuitively quite natural, because we want a round shape and bending non-triangular faces by pulling outward their diagonals does not increase the number of vertices. We shall prove Theorem 1 after Lemma 3. Here we quote a paragraph on Theorem 1 in page 58 of Fejes Tóth [5] (see also $[4,7])$.

Greifen wir um dies einzusehen eine beliebige Ecke $E$ des als extremal vorausgesetzten Polyeders heraus und bewegen es so, dass erstens der Inhalt, zweitens die Oberfläche der kleinsten konvexen Hülle $\mathbb{H}$ von E und der Übrigen Ecken des Polyeders konstant bleiben. Im ersten Fall durchläuft E den Rand eines konvexen Polyeders $\mathbb{P}$, im zweiten Fall dagegen den Rand eines singularitätenfreien Eikörpers $\mathbb{E}$, der im Falle eines Extremalen Polyeders offenkundig keinen Punkt ausserhalb $\mathbb{P}$ haben kann. Währe nun E die Ecke einer mehr als dreiseitigen Fläche des ursprünglichen Polyeders, so liege E-wie eine einfache Überlegung zeigt- auf einer Kante von $\mathbb{P}$. Mithin könnte $\mathbb{P}$ nicht die singularitätenfreie Fläche $\mathbb{E}$ enthalten.

(English Translation) In order to see this, let us take an arbitrary vertex $E$ of the polyhedron, which is supposed to be extremal. We can move it keeping firstly the volume, and secondly the surface area, of the smallest convex hull $\mathbb{H}$ of $E$ and the remaining vertices of the polyhedron. In the first case, $E$ passes through the boundary of a convex polyhedron $\mathbb{P}$, and in the second case, it passes the boundary of a singularity-free egg-shaped body $\mathbb{E}$, which is obviously not a point outside $\mathbb{P}$ in the case of an extremal polyhedron. If $E$ is the vertex of a more than three-sided face of the polyhedron, then, it is on an edge of $\mathbb{P}$ by a simple discussion. Thus $\mathbb{P}$ cannot contain the singularity-free surface $\mathbb{E}$.

Let us try to understand this description and then show where the gap is. A point $\mathbf{x}$ in a convex set $X$ is visible from a point $\mathbf{y} \in \mathbb{R}^{3} \backslash X$, if the segment $[\mathbf{x}, \mathbf{y}]$ intersects $X$ only at $\mathbf{x}$. A subset $V$ of $X$ is visible from $\mathbf{y}$ if each element of $V$ is visible from $\mathbf{y}$. A face plane of a polyhedron $X$ is a hyperplane containing a codimension one face of $X$. Let us fix $p_{1}, p_{2}, \ldots$, $p_{n-1}$. Define

$$
C=\left\{\mathbf{v} \in \mathbb{R}^{3} \mid V_{3}\left(\Delta\left(p_{1}, \ldots, p_{n-1}, \mathbf{v}\right)\right) \leq v\right\}
$$

and

$$
S=\left\{\mathbf{v} \in \mathbb{R}^{3} \mid A_{3}\left(\Delta\left(p_{1}, \ldots, p_{n-1}, \mathbf{v}\right)\right) \leq h\right\}
$$

with $\quad v>V_{3}\left(\Delta\left(p_{1}, \ldots, p_{n-1}\right)\right) \quad$ and $\quad h>A_{3}\left(\Delta\left(p_{1}, \ldots, p_{n-1}\right)\right)$. Clearly $V_{3}\left(\Delta\left(p_{1}, \ldots, p_{n-1}, \mathbf{v}\right)\right)$ and $A_{3}\left(\Delta\left(p_{1}, \ldots, p_{n-1}, \mathbf{v}\right)\right)$ are continuous functions of v. The boundary $\partial(C)$ is a contour of the volume function of the convex hull of $\mathbf{v}$ and visible faces from $\mathbf{v}$ of $\Delta\left(p_{1}, \ldots, p_{n-1}\right)$. Visible faces change when and only when $\mathbf{v}$ passes a face plane of $\Delta\left(p_{1}, \ldots, p_{n-1}\right)$ and that makes a visible 
face $F$ into a non-visible one, or the other way round. Note that this change happens only when a non-triangular face with a vertex $\mathbf{v}$ appears in $\Delta\left(p_{1}, \ldots\right.$, $\left.p_{n-1}, \mathbf{v}\right)$. This volume is an affine function on the coordinates of $\mathbf{v}$ determined by visible faces from $\mathbf{v}$. Therefore $C$ is the intersection of half-spaces defined by visible faces, i.e., a convex polyhedron. If there exists a non-triangular face, then $\mathbf{v}$ must be on the edge of $\partial(C)$.

The surface $\partial(S)$ is determined by visible edges from $\mathbf{v}$ which contribute the surface of the convex hull. Locally $\partial(S)$ is defined as a contour of sum of square roots of quadratic polynomials of its coordinates, which implies that $\partial(S)$ is piecewise smooth. While $\mathbf{v}$ moves around, visible edges will switch to new ones when the visible faces change. Note that this change happens when $\mathbf{v}$ is on a face plane of $\Delta\left(p_{1}, \ldots, p_{n-1}\right)$. Assume that $\Delta_{n}=\Delta\left(p_{1}, \ldots, p_{n-1}, p_{n}\right)$ is a minimum $n$-hedron and $p_{n}$ is a vertex of a non-triangular face. Then $p_{n}$ must be on the edge of $\partial(C)$. Put $v=V_{3}\left(\Delta_{n}\right)$ and $h=A_{3}\left(\Delta_{n}\right)$. If $p_{n}$ is a totally differentiable point of $S$, then the surface $\partial(S)$ penetrates $\partial(C)$ and we must have a point of $\partial(S)$ outside $C$, which contradicts the minimality of $\Delta\left(p_{1}, \ldots, p_{n}\right)$. Therefore in this view, if $\partial(S)$ is totally differentiable everywhere, the proof is done (see [2, Lemma 4.4]).

This idea is very insightful but does not work as it is. Here is a counter example for $n=5$ such that $\partial(S)$ has a singular point.

ExAmple 1. Let $p_{1}=(0,1,0), p_{2}=(0,0,0), p_{3}=(1,0,0), p_{4}=(0,0,1)$. The surface $S$ with $h=4$ consists of 14 algebraic surfaces, and the black point $(0,2,0)$ is a common point of four surfaces depicted in Figure 1 . We claim that this point $(0,2,0)$ is singular. Indeed it is on the boundary of four surfaces

$$
\begin{gathered}
\sqrt{2 x^{2}+(y+z-1)^{2}}+\sqrt{(x+z-1)^{2}+2 y^{2}} \\
+\sqrt{(x+y-1)^{2}+2 z^{2}}+3=8 \\
\sqrt{x^{2}+y^{2}}+\sqrt{(x+z-1)^{2}+2 y^{2}}+\sqrt{y^{2}+z^{2}}+1=8, \\
\sqrt{2 x^{2}+(y+z-1)^{2}}+\sqrt{x^{2}+z^{2}}+\sqrt{(x+z-1)^{2}+2 y^{2}}+\sqrt{y^{2}+z^{2}}+2=8, \\
\sqrt{x^{2}+y^{2}}+\sqrt{x^{2}+z^{2}}+\sqrt{(x+z-1)^{2}+2 y^{2}}+\sqrt{(x+y-1)^{2}+2 z^{2}}+2=8,
\end{gathered}
$$

whose domains and visible edges which contribute to $A_{3}\left(\Delta\left(p_{1}, p_{2}, p_{3}, p_{4}, \mathbf{v}\right)\right)$ are

$$
\begin{array}{ll}
\{(x, y, z) \mid x \geq 0, y \geq 0, z \geq 0, x+y+z \geq 1\}, & \left\{\left\{p_{4}, p_{1}\right\},\left\{p_{3}, p_{4}\right\},\left\{p_{1}, p_{3}\right\}\right\} \\
\{(x, y, z) \mid x \leq 0, y \geq 0, z \leq 0, x+y+z \geq 1\}, & \left\{\left\{p_{2}, p_{4}\right\},\left\{p_{4}, p_{3}\right\},\left\{p_{3}, p_{2}\right\}\right\}
\end{array}
$$




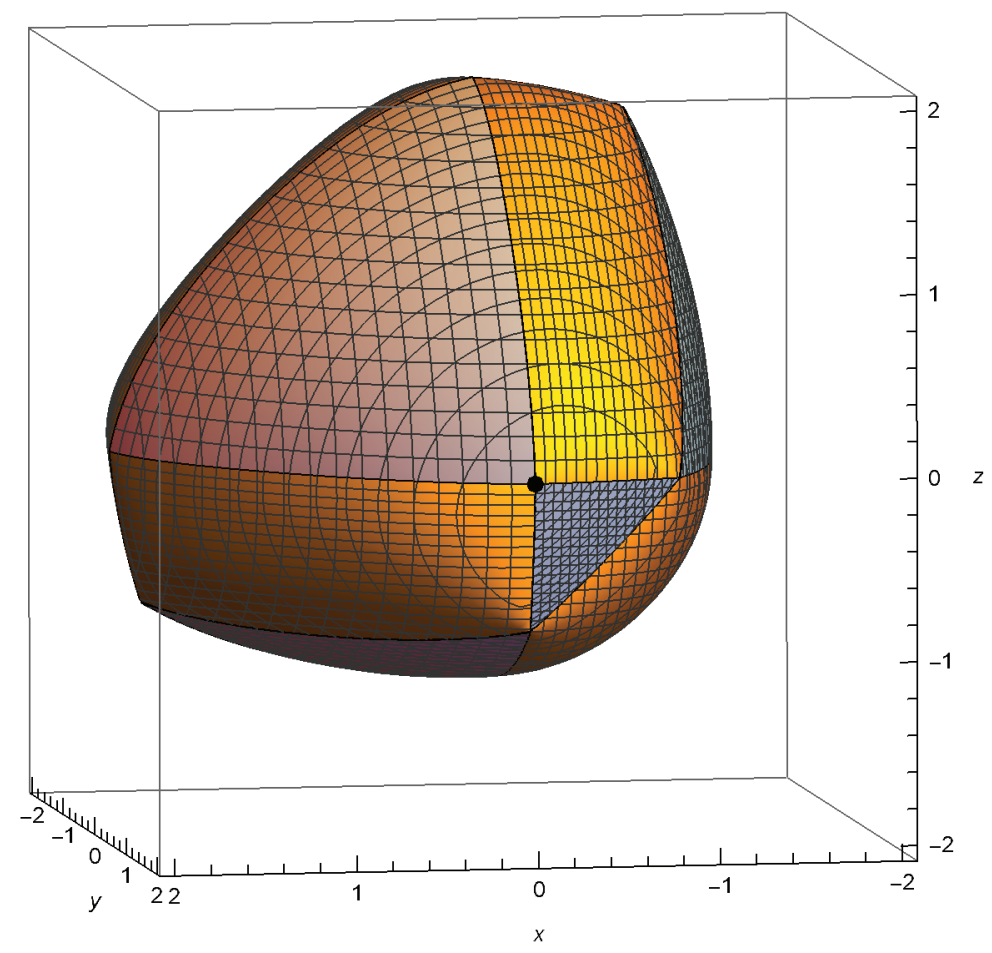

Fig. 1. The surface $\partial(S)$ with singular points

$$
\begin{gathered}
\{(x, y, z) \mid x \geq 0, y \geq 0, z \leq 0, x+y+z \geq 1\}, \\
\left\{\left\{p_{4}, p_{1}\right\},\left\{p_{2}, p_{1}\right\},\left\{p_{3}, p_{4}\right\},\left\{p_{3}, p_{2}\right\}\right\}, \\
\{(x, y, z) \mid x \leq 0, y \geq 0, z \geq 0, x+y+z \geq 1\}, \\
\left\{\left\{p_{2}, p_{4}\right\},\left\{p_{2}, p_{1}\right\},\left\{p_{3}, p_{4}\right\},\left\{p_{3}, p_{1}\right\}\right\},
\end{gathered}
$$

respectively. For example, (6) follows from

$$
\begin{aligned}
& V_{2}\left(p_{4}, p_{1}, \mathbf{v}\right)+V_{2}\left(p_{3}, p_{4}, \mathbf{v}\right)+V_{2}\left(p_{1}, p_{3}, \mathbf{v}\right) \\
& \quad+V_{2}\left(p_{1}, p_{2}, p_{3}\right)+V_{2}\left(p_{1}, p_{2}, p_{4}\right)+V_{2}\left(p_{2}, p_{3}, p_{4}\right)=4 .
\end{aligned}
$$

For the first two surfaces, outer normals at $(0,2,0)$ approaching from the corresponding domains are $(1,5,1),(-1,10,-1)$, which are mutually inconsistent, and the tangent plane at $(0,2,0)$ cannot be defined. For the remaining two surfaces, the situation is worse that $(0,2,0)$ becomes a singular point by the effect of the term $\sqrt{x^{2}+z^{2}}$, whose partial derivatives on $x, z$ vary by the ratio $x: z$. 
We shall see in Lemma 3 that this type of singularity never vanishes regardless of the choice of $h$.

A polyhedral cell is a closed convex set with a non-empty interior whose boundary consists of finite number of convex subsets of hyperplanes of codimension 1. Hereafter we use a partition of $\mathbb{R}^{3}$ into polyhedral cells by face planes $W_{i}$ of a convex hull $\Delta$. For $\mathbf{v} \in \mathbb{R}^{3} \backslash \Delta$, consider a plane $W$ separating $\Delta$ and $v$. Then the union of visible faces from $\mathbf{v}$ is homeomorphically mapped to a figure of $W$ by a projection sending a point $y$ on the union to the point $y^{\prime} \in W$ if $y, y^{\prime}, \mathbf{v}$ are collinear. We say that the resulting figure is the planar projection. Planar projections are affine equivalent under the change of separating planes. We prepare an important property of visibility.

Lemma 2. The planar projection of the union of visible faces $\left\{Q_{i}\right\}_{i=1}^{k}$ from $\mathbf{v}$ is convex.

Proof. This follows immediately from the convexity of $\Delta$.

We first confirm that Fejes Tóth's idea is almost valid, but the surface $\partial(S)$ must have a singular point.

Lemma 3. Assume that $\Delta\left(p_{1}, \ldots, p_{n-1}\right)$ is non-degenerate and fix a positive constant $h>A_{3}\left(\Delta\left(p_{1}, p_{2}, \ldots, p_{n-1}\right)\right)$. The surface

$$
\partial(S)=\left\{\mathbf{v} \in \mathbb{R}^{3} \mid A_{3}\left(\Delta\left(p_{1}, \ldots, p_{n-1}, \mathbf{v}\right)\right)=h\right\}
$$

is totally differentiable except at most 2 e points, where $e$ is the number of edges of $\Delta\left(p_{1}, \ldots, p_{n-1}\right)$. The surface $\partial(S)$ is not totally differentiable at $\mathbf{v} \in \partial(S)$ if and only if the prolongation of an edge of $\Delta\left(p_{1}, \ldots, p_{n-1}\right)$ penetrates $\mathbf{v}$.

Proof. We prove that $\partial(S)$ is totally differentiable at a switching point contained in exactly one face plane of $\Delta\left(p_{1}, \ldots, p_{n-1}\right)$. This switching occurs at several contiguous edges forming a broken line which are the edges of the changing face. A crucial point is that the initial and the final vertices of this broken line do not change by the switching. For example, consider a (planar) convex quadrangle $K L M N$ with $K=p_{1}, L=p_{2}, M=p_{3}$. This could be divided into two triangles in two different ways, like $K L N, L M N$ or $K L M$, $K N M$. Edge switching occurs when $\mathbf{v}$ passes transversally through $N$. First the area of triangles $K L \mathbf{v}$ and $L M \mathbf{v}$ contributes to $A_{3}$, and later, the triangles $K L M$ and $K \mathbf{v} M$ do. In this case, the related edges are $K L, L M$ at the beginning and are switched to $K M$ (see Figure 2).

To see that $\partial(S)$ admits a tangent plane at the switching point $\mathbf{v}$, assume that the switching of edges happens on the plane $z=0$ to simplify the 


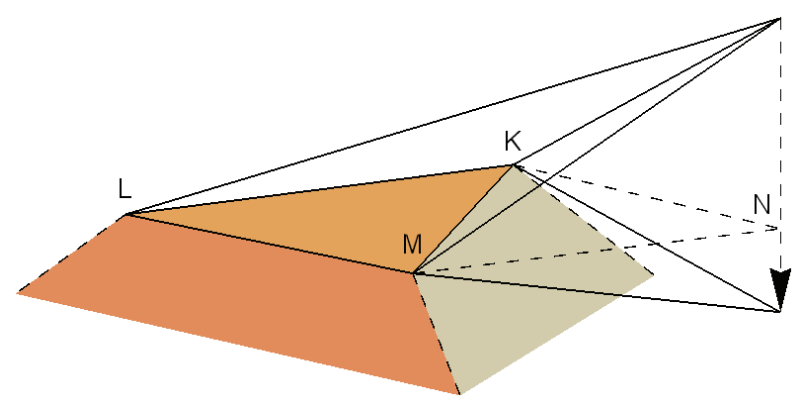

Fig. 2. Switching visible edges

computation. Let

$$
\left(a_{i}, b_{i}, 0\right),\left(a_{i+1}, b_{i+1}, 0\right) \quad(i=1, \ldots, k-1)
$$

be the end points of the switching edges, and $(x, y, z) \in \partial(S)$. Then the surface $\partial(S)$ on one side of the switching plane is defined locally by an equation of the form $f(x, y, z)+m(x, y, z)=h$ :

$$
f=\sum_{i=1}^{k-1} \frac{\sqrt{\left(\left(b_{i}-b_{i+1}\right)^{2}+\left(a_{i}-a_{i+1}\right)^{2}\right) z^{2}+\left(\left(b_{i}-b_{i+1}\right) x-\left(a_{i}-a_{i+1}\right) y+a_{i} b_{i+1}-b_{i} a_{i+1}\right)^{2}}}{2},
$$

where $m(x, y, z)$ is the contribution from non-switching edges. The equation and the value $k$ change when $v$ passes the switching plane. At a switching point $\left(x_{0}, y_{0}, 0\right)$, we have

$$
\begin{aligned}
& \frac{\partial(f+m)}{\partial x}=\sum_{i=1}^{k-1} \frac{b_{i}-b_{i+1}}{2} \frac{\left(b_{i}-b_{i+1}\right) x_{0}-\left(a_{i}-a_{i+1}\right) y_{0}+a_{i} b_{i+1}-b_{i} a_{i+1}}{\sqrt{\left(\left(b_{i}-b_{i+1}\right) x_{0}-\left(a_{i}-a_{i+1}\right) y_{0}+a_{i} b_{i+1}-b_{i} a_{i+1}\right)^{2}}}+\frac{\partial m}{\partial x}, \\
& \frac{\partial(f+m)}{\partial y}=\sum_{i=1}^{k-1} \frac{a_{i+1}-a_{i}}{2} \frac{\left(b_{i}-b_{i+1}\right) x_{0}-\left(a_{i}-a_{i+1}\right) y_{0}+a_{i} b_{i+1}-b_{i} a_{i+1}}{\sqrt{\left(\left(b_{i}-b_{i+1}\right) x_{0}-\left(a_{i}-a_{i+1}\right) y_{0}+a_{i} b_{i+1}-b_{i} a_{i+1}\right)^{2}}}+\frac{\partial m}{\partial y}
\end{aligned}
$$

and

$$
\frac{\partial(f+m)}{\partial z}=\frac{\partial m}{\partial z}
$$

From Lemma 2, we see that $\left(a_{i}, b_{i}, 0\right)(i=1, \ldots, k)$ and $\left(x_{0}, y_{0}, 0\right)$ form vertices of a planar convex $(k+1)$-gon. Consequently

$$
\left(b_{i}-b_{i+1}\right) x_{0}-\left(a_{i}-a_{i+1}\right) y_{0}+a_{i} b_{i+1}-b_{i} a_{i+1}
$$


have the same sign for all $i$, and the normal vector of $\partial(S)$ at $\left(x_{0}, y_{0}, 0\right)$ is $\left(b_{1}-b_{k}, a_{k}-a_{1}, 0\right) \pm 2 m^{\prime}$, where $m^{\prime}$ is the contribution from $m(x, y, z)$. The vector $\left(b_{1}-b_{k}, a_{k}-a_{1}, 0\right)$ is orthogonal to the segment joining two end points of the switching broken line, i.e., the segment between $\left(a_{1}, b_{1}, 0\right)$ and $\left(a_{k}, b_{k}, 0\right)$. As these two end points are invariant under switching, even at the switching point $\left(x_{0}, y_{0}, 0\right)$ the tangent plane is well-defined. Therefore the surface $\partial(S)$ is totally differentiable at any switching point contained in exactly one face plane of $\Delta\left(p_{1}, \ldots, p_{n-1}\right)$.

Let us study the possible singularities. The switching points lying on two or more face planes are on the intersection lines of face planes. Assume that a point $\mathbf{v}$ is lying on face planes $W_{j}(j=1, \ldots, \ell)$ with $\ell \geq 2$. The face plane $W_{j}$ induces switching of edges $e_{1}^{(j)}, \ldots, e_{k_{j}}^{(j)}\left(k_{j} \geq 2\right)$ to $e^{\prime(j)}$ or the other way round. ${ }^{2}$ Of course $e^{\prime(j)}(j=1, \ldots, \ell)$ are distinct. If the set of edges $E_{j}=$ $\left\{e_{i}^{(j)} \mid i=1, \ldots, k_{j}\right\}$ are mutually disjoint for $j=1, \ldots, \ell$, then $\partial(S)$ is totally differentiable at $\mathbf{v}$ by the same proof. The singularity happens only when there exist $j_{1} \neq j_{2}$ for which $E_{j_{1}} \cap E_{j_{2}} \neq \varnothing$. We claim that this is also sufficient. In fact, such an intersection must be a single edge and its prolongation must pass through the point $\mathbf{v}$. This means that around $\mathbf{v}$, there is a polyhedral cell $K$ such that if $\mathbf{u} \in K$ then there is a visible edge from u penetrating $\mathbf{v}$, that contributes the sum of the surface area of $\Delta\left(p_{1}, \ldots, p_{n-1}, \mathbf{u}\right)$. This contribution is the square root of a positive semi-definite quadratic form over three variables $x-a, y-b, z-c$ with $\mathbf{v}=(a, b, c)$, which vanishes ${ }^{3}$ when and only when $\mathbf{u}$ is on the line passing the visible edge penetrating $\mathbf{v}$. Such a term corresponds exactly to two visible faces and gives a conic singularity. In most cases, such a term is unique and it produces a singularity at v. For special cases, there may be several terms of this type, so that each term vanishes on different prolongations of edges of $\Delta\left(p_{1}, \ldots, p_{n-1}\right)$ penetrating $\mathbf{v}$. If there are more than one such terms, then all partial derivatives of the terms at $\mathbf{v}$ with respect to variables $x-a, y-b, z-c$ are zero. Therefore the singularity at $\mathbf{v}$ becomes removable after the summation only when this algebraic function becomes locally a constant. However then the sum is constant everywhere, by algebraicity. This cannot happen because every term diverges to positive infinity by taking limit in all directions except the vanishing line. Therefore $\partial(S)$ cannot be totally differentiable at $\mathbf{v}$. This shows the claim and finishes the proof.

\footnotetext{
${ }^{2}$ In the above proof, the face plane is $z=0$, and $e_{i}^{(j)}=\left[\left(a_{i}, b_{i}, 0\right),\left(a_{i+1}, b_{i+1}, 0\right)\right], e^{\prime(j)}=$ $\left[\left(a_{1}, b_{1}, 0\right),\left(a_{k}, b_{k}, 0\right)\right]$.

${ }^{3}$ In Example 1, this is the term $\sqrt{x^{2}+z^{2}}$, which vanishes on the line through the edge $\left\{p_{1}, p_{2}\right\}$.
} 
Proof of TheOrem 1. If there exists a non-triangular face and $p_{n}$ is a vertex on this face, then $\mathbf{v}=p_{n}$ must be on an edge of $\partial(C)$. Here $C$ and $S$ are defined by (4) and (5) respectively. By the discussion before Example 1, if $\mathbf{v}=p_{n}$ is non-singular then we get a contradiction. Let $\mathbf{v}=p_{n}$ be one of the singular points of $\partial(S)$ in Lemma 3 . Then there is an edge of $\Delta\left(p_{1}, \ldots, p_{n-1}\right)$ penetrating $p_{n}$. However this implies that one of the vertices of $\Delta\left(p_{1}, \ldots\right.$, $\left.p_{n-1}\right)$ is in the relative interior of an edge of $\Delta\left(p_{1}, \ldots, p_{n-1}, p_{n}\right)$. In this case the number of vertices of $\Delta\left(p_{1}, \ldots, p_{n}\right)$ is less than $n$. Since $\Delta\left(p_{1}, \ldots, p_{n-1}, p_{n}\right)$ is a minimum $n$-hedron, this does not happen by Proposition 2, giving a contradiction for this case.

It is possible to give a geometric (but more technical) alternative proof of Theorem 1 without using the last characterization of the singularity in Lemma 3. We give a rough sketch of it. The singular point of $\mathbf{v}=p_{n}$ of $\partial(S)$ in the above proof is defined by piecewise smooth surfaces. Take polyhedral cells $K_{1}, K_{2}$ defined by the face planes $W_{j}(j=1, \ldots, \ell)$ with the maximum and minimum number of visible faces. It is clear that on $K_{i}(i=1,2)$ we see no visible edges passing $\mathbf{v}$, and therefore the tangent planes approaching from $K_{i}$ are well defined. This tangent plane must coincide with the corresponding face planes of $\partial(C)$ (otherwise one can prolong a tangent plane which penetrates $\partial(C)$ giving a smaller $A_{3}(\Delta) / V_{3}(\Delta)^{2 / 3}$ by a non-convex $\left.\Delta\right)$. On the other hand, approaching to $\mathbf{v}$ from other polyhedral cells surrounding $\mathbf{v}$, the point $\mathbf{v}$ is singular. Partial derivatives of the singular terms which appear in this intermediate terms are the function on the ratio of $x-a: y-b: z-c$ with $\mathbf{v}=$ $(a, b, c)$. Take a slice of $\partial(S)$ by a plane which passes an inner point of $C$ close to $\mathbf{v}$ and intersects all $W_{j}$. This gives a piecewise smooth planar curve that has two 'almost' linear parts and other parts with positive curvature. Shifting the slice plane parallel and closer to $p$, the shape converges to a single curve up to similitude, which encircles a convex planar region. On the other hand, since tangent planes exist within $K_{i}$, the parts of the curve in $K_{i}$ converge to line segments. Recalling $S \subset C$, this causes an inconsistency at their end points.

\section{One vertex deformation is impossible}

In this section, we further develop the idea of Fejes Tóth and show that the minimum $n$-hedron does not allow a deformation of a single vertex.

Let $X$ be a convex set in $\mathbb{R}^{d}$. A function $F: X \rightarrow \mathbb{R}$ is convex if for any $\mathbf{u}, \mathbf{v} \in X$ and any $\lambda \in[0,1]$, we have

$$
F((1-\lambda) \mathbf{u}+\lambda \mathbf{v}) \leq(1-\lambda) F(\mathbf{u})+\lambda F(\mathbf{v}) .
$$


It is strictly convex if for any $\mathbf{u}, \mathbf{v} \in X$ with $\mathbf{u} \neq \mathbf{v}$ and any $\lambda \in(0,1)$,

$$
F((1-\lambda) \mathbf{u}+\lambda \mathbf{v})<(1-\lambda) F(\mathbf{u})+\lambda F(\mathbf{v}) .
$$

Take a convex subset $Y \subset X$. If $F: X \rightarrow \mathbb{R}$ is convex and the equality of (7) with $\lambda \in(0,1)$ holds only when $\mathbf{u}, \mathbf{v} \in Y$, then we say $X$ is strictly convex except $Y$.

The next lemma gives a method to paste together convex functions defined in polyhedral cells to obtain a global convex function. Related general criteria are found in [1] using convex analysis.

LEMMA 4. Let $\mathbb{R}^{d}$ be partitioned into a finite number of polyhedral cells $\left\{D_{i}\right\}$ whose interiors are disjoint. Let $Z$ be the set of points of $\mathbb{R}^{d}$ that belong to more than two $D_{i}$. Assume that $F_{i}$ is a convex function on $D_{i}$ so that $F_{i}(\mathbf{v})=$ $F_{j}(\mathbf{v})$ holds for each $\mathbf{v} \in D_{i} \cap D_{j}$. Then the function $F: \mathbb{R}^{d} \rightarrow \mathbb{R}$ is naturally defined by the values of $F_{i}$. We see that $F$ is convex if and only if the following condition holds

- If $\mathbf{v} \in\left(D_{i} \cap D_{j}\right) \backslash Z, \mathbf{v}-\omega \in D_{i}$ and $\mathbf{v}+\omega \in D_{j}$ for $\omega \neq 0$, then there exists a positive $t \in(0,1)$ such that $F(\mathbf{v}) \leq\left(F_{i}(\mathbf{v}-t \omega)+F_{j}(\mathbf{v}+t \omega)\right) / 2$.

If each $F_{i}$ is strictly convex, then $F$ is strictly convex.

Noting that $\omega$ can be chosen arbitrarily small, the condition in Lemma 4 is a local property around $D_{i} \cap D_{j}$.

Proof. The condition is clearly necessary. We prove the sufficiency. Note that since $Z$ is of dimension $d-2$ or less, if the condition is valid for $\mathbf{v} \in\left(D_{i} \cap D_{j}\right) \backslash Z$ then it is also valid for $D_{i} \cap D_{j}$ by continuity of convex functions. Let us show the simplest case that $\mathbb{R}^{d}=D_{1} \cup D_{2}$ and $D_{1} \cap D_{2}$ is a hyperplane. Take $\mathbf{x} \in D_{1}, \mathbf{v} \in D_{2}$ and find $\mathbf{w} \in[\mathbf{x}, \mathbf{v}] \cap D_{1} \cap D_{2}$. By the assumption, if $0 \neq \omega$ is a positive multiple of $\mathbf{v}-\mathbf{x}$, there exists $t>0$ such that

$$
F(\mathbf{w}) \leq \frac{1}{2}\left(F_{1}(\mathbf{w}-t \omega)+F_{2}(\mathbf{w}-t \omega)\right)
$$

where $\mathbf{w}-t \omega \in(\mathbf{x}, \mathbf{w})$ and $\mathbf{w}+t \omega \in(\mathbf{w}, \mathbf{v})$. Therefore we find $\mu_{1}, \mu_{2} \in(0,1)$ such that $\mathbf{w}-t \omega=\left(1-\mu_{1}\right) \mathbf{x}+\mu_{1} \mathbf{w}$ and $\mathbf{w}+t \omega=\left(1-\mu_{2}\right) \mathbf{w}+\mu_{2} \mathbf{v}$. Using convexity of $F_{i}$, we have

$$
F_{1}(\mathbf{w}-t \omega) \leq\left(1-\mu_{1}\right) F_{1}(\mathbf{x})+\mu_{1} F(\mathbf{w})
$$

and

$$
F_{2}(\mathbf{w}+t \omega) \leq\left(1-\mu_{2}\right) F(\mathbf{w})+\mu_{2} F_{2}(\mathbf{v})
$$


Using (8), (9) and (10), we deduce

$$
F(\mathbf{w}) \leq \frac{1-\mu_{1}}{1-\mu_{1}+\mu_{2}} F_{1}(\mathbf{x})+\frac{\mu_{2}}{1-\mu_{1}+\mu_{2}} F_{2}(\mathbf{v}) .
$$

Because we can take arbitrarily small $t$, the required convexity inequality holds for all $\mathbf{x} \in D_{1}, \mathbf{v} \in D_{2}$ and $\mathbf{w} \in(\mathbf{x}, \mathbf{v}) \cap D_{1} \cap D_{2}$. Take $\mathbf{u}, \mathbf{x} \in D_{1}, \mathbf{w} \in D_{1} \cap D_{2}$, $\mathbf{v} \in D_{2}$ so that $\mathbf{x}, \mathbf{w}$ are within the open segment $(\mathbf{u}, \mathbf{v})$. Take $\lambda, \mu \in(0,1)$ such that $\mathbf{w}=(1-\lambda) \mathbf{x}+\lambda \mathbf{v}$ and $\mathbf{x}=(1-\mu) \mathbf{u}+\mu \mathbf{w}$. By the above discussion, we have

$$
F(\mathbf{w}) \leq(1-\lambda) F_{1}(\mathbf{x})+\lambda F_{2}(\mathbf{v}) .
$$

By the convexity of $F_{1}$,

$$
F_{1}(\mathbf{x}) \leq(1-\mu) F_{1}(\mathbf{u})+\mu F(\mathbf{w})
$$

From (11) and (12), we obtain

$$
F_{1}(\mathbf{x}) \leq \frac{1-\mu}{1-\mu+\mu \lambda} F_{1}(\mathbf{u})+\frac{\mu \lambda}{1-\mu+\mu \lambda} F_{2}(\mathbf{v}) .
$$

Summing up, we know that any pair of two points $\mathbf{u} \in D_{1}$ and $\mathbf{v} \in D_{2}$, the required convexity inequality is valid for any point $\mathbf{x} \in(\mathbf{u}, \mathbf{v})$. Therefore we can merge domains of convexity and the proof for the case $\mathbb{R}^{d}=D_{1} \cup D_{2}$ is finished. If each $F_{i}$ is strictly convex, then the resulting inequality is strict. One can easily extend this discussion to the general case. We simply repeat the merging process for adjacent domains sharing a codimension one face. The set $Z$ does not disturb this merging process because $\left\{D_{i}\right\}$ are chain connected by the adjacency relation induced by codimension one faces.

A convex body $X$ is strictly convex, if $\mathbf{x}, \mathbf{y} \in X$ with $\mathbf{x} \neq \mathbf{y}$, then $(1-\lambda) \mathbf{x}+\lambda \mathbf{y} \in \operatorname{Inn}(X)$ for $\lambda \in(0,1)$, where $\operatorname{Inn}(X)$ is the interior of $X$. It is easy to see that a non-empty set of the form $\{\mathbf{v} \mid F(\mathbf{v}) \leq h\}$ for some $h>0$ is strictly convex if $F$ is strictly convex except $Y$ with a convex $Y \subset \operatorname{Inn}(X)$.

THEOREM 2. $S$ is strictly convex.

Proof. As in the proof of Theorem 1, considering $p_{n}$ as a variable $\mathbf{v}$, the surface $\partial(S)$ is a contour of the sum of $V_{2}\left(q_{i}, q_{i+1}, \mathbf{v}\right)$, where $\left[q_{i}, q_{i+1}\right]$ $(i=0, \ldots, \ell-1)$ are the related visible edges. Here $q_{i} \in\left\{p_{1}, \ldots, p_{n-1}\right\}$ and the index $i$ is considered modulo $\ell$. The hyperplanes which contain a face of $\Delta\left(p_{1}, \ldots, p_{n-1}\right)$ gives a partition $\left\{D_{i}\right\}_{i \geq 1}$ of $\mathbb{R}^{d}$ into a finite number of polyhedral cells, and the set of visible faces is invariant within each $D_{i}$ outside $\Delta\left(p_{1}, \ldots, p_{n-1}\right)$. Let $F_{i}$ be the function $A_{3}\left(p_{1}, \ldots, p_{n-1}, \mathbf{v}\right)$ restricted to $D_{i}$, and define a constant function $F_{0}(\mathbf{v})=A_{3}\left(\Delta\left(p_{1}, \ldots, p_{n-1}\right)\right)$ for $\mathbf{v} \in D_{0}:=\Delta\left(p_{1}, \ldots\right.$, 
$\left.p_{n-1}\right)$. Then $F_{i}(\mathbf{v})=F_{j}(\mathbf{v})$ for $\mathbf{v} \in D_{i} \cap D_{j}$ is clear. Let $Z$ be as in Lemma 4, which is a finite set of $\mathbb{R}^{3}$. We claim that the condition of Lemma 4 is also satisfied. Indeed in the same way as in the proof of Theorem $1, A_{3}\left(\Delta\left(p_{1}, \ldots\right.\right.$, $\left.\left.p_{n-1}, \mathbf{v}\right)\right)$ is totally differentiable at $\mathbf{v} \in\left(D_{i} \cap D_{j}\right) \backslash Z$ with $1 \leq i<j$, i.e.,

$$
F(\mathbf{u})=F(\mathbf{v})+\nabla F(\mathbf{v}) \cdot(\mathbf{u}-\mathbf{v})+o(\|\mathbf{u}-\mathbf{v}\|)
$$

with $\nabla F(\mathbf{v}) \neq(0,0,0)$. Take $\omega \neq 0$ such that $\mathbf{v}-\omega \in D_{i}, \mathbf{v}+\omega \in D_{j}$. If $F_{i}\left(\mathbf{v}-t_{0} \omega\right)<F(\mathbf{v})+\nabla F(\mathbf{v}) \cdot\left(-t_{0} \omega\right)$ for some $t_{0} \in(0,1)$, then $\left(F_{i}(\mathbf{v}-t \omega)-\right.$ $\left.F_{i}(\mathbf{v})\right) / t \leq\left(F_{i}\left(\mathbf{v}-t_{0} \omega\right)-F_{i}(\mathbf{v})\right) / t_{0} \leq M$ for all $t \in\left(0, t_{0}\right)$ with a constant $M<$ $\nabla F(\mathbf{v}) \cdot(-\omega)$ by convexity of $F_{i}$. This contradicts (13) and we see $F_{i}\left(\mathbf{v}-t_{0} \omega\right)$ $\geq F(\mathbf{v})+\nabla F(\mathbf{v}) \cdot\left(-t_{0} \omega\right)$. In the same way, we have $F_{j}\left(\mathbf{v}+t_{0} \omega\right) \geq F(\mathbf{v})+$ $\nabla F(\mathbf{v}) \cdot\left(t_{0} \omega\right)$ and thus $F(\mathbf{v}) \leq\left(F_{i}\left(\mathbf{v}-t_{0} \omega\right)+F_{j}\left(\mathbf{v}+t_{0} \omega\right)\right) / 2$. For $\mathbf{v} \in D_{0} \cap D_{j}$ with $j \geq 1$, the condition is trivial because $A_{3}\left(\Delta\left(p_{1}, \ldots, p_{n-1}, \mathbf{v}\right)\right) \geq$ $A_{3}\left(\Delta\left(p_{1}, \ldots, p_{n-1}\right)\right)$. It remains to show that each $F_{i}(i \geq 1)$ is strictly convex to apply Lemma 4 .

Clearly $\ell \geq 3$. We claim that $V_{2}\left(q_{i}, q_{i+1}, \mathbf{x}\right)$ is a convex function. Indeed, consider a plane $P_{i}$ passing $q_{i}$ perpendicular to $\left[q_{i}, q_{i+1}\right]$ and the orthogonal projection $g$ to $P_{i}$. Then we have $V_{2}\left(q_{i}, q_{i+1}, \mathbf{x}\right)=\left\|g(\mathbf{x})-q_{i}\right\|\left\|q_{i+1}-q_{i}\right\| /$ 2. Since $g$ is linear and $g\left(q_{i}\right)=q_{i}$, the triangle inequality implies

$$
\left\|g((1-\lambda) \mathbf{x}+\lambda \mathbf{y})-q_{i}\right\| \leq(1-\lambda)\left\|g(\mathbf{x})-q_{i}\right\|+\lambda\left\|g(\mathbf{y})-q_{i}\right\|
$$

for $\lambda \in[0,1]$, which proves the claim. As the sum of convex function is convex, we know $\sum_{i=0}^{\ell-1} V_{2}\left(q_{i}, q_{i+1}, \mathbf{x}\right)$ and $F$ are convex. The equality for $\lambda \in(0,1)$ in (14) occurs only if $g(\mathbf{x})-q_{i}$ and $g(\mathbf{y})-q_{i}$ are linearly dependent. This happens only when $\mathbf{x}, \mathbf{y}, q_{i}, q_{i+1}$ are in the same plane. However we can find an index $i$ such that $\mathbf{x}, \mathbf{y}, q_{i}, q_{i+1}$ are not in the same plane. Indeed, by our implicit assumption on visibility, the $\ell+2$ points $\{\mathbf{x}, \mathbf{y}\} \cup\left\{q_{i} \mid i=0, \ldots\right.$, $\ell-1\}$ cannot be in the same plane. Therefore, we always have

$$
\sum_{i=0}^{\ell-1} V_{2}\left(q_{i}, q_{i+1},(1-\lambda) \mathbf{x}+\lambda \mathbf{y}\right)<(1-\lambda) \sum_{i=0}^{\ell-1} V_{2}\left(q_{i}, q_{i+1}, \mathbf{x}\right)+\lambda \sum_{i=0}^{\ell-1} V_{2}\left(q_{i}, q_{i+1}, \mathbf{x}\right)
$$

for $\lambda \in(0,1)$. This proves that $F$ is strictly convex except $\Delta\left(p_{1}, \ldots, p_{n-1}\right)$. Since $S=\left\{\mathbf{v} \in \mathbb{R}^{3} \mid F(\mathbf{v}) \leq h\right\}$ for some $h>0$, we have shown the theorem.

Corollary 1. A minimum n-hedron $\Delta_{n}=\Delta\left(p_{1}, \ldots, p_{n}\right)$ does not allow deformation of a single vertex, i.e., there exists a positive $r$ so that if $\Delta\left(p_{1}, \ldots\right.$, $\left.p_{n-1}, \mathbf{x}\right)$ is a minimum n-hedron with $\left\|\mathbf{x}-p_{n}\right\|<r$, then $\mathbf{x}=p_{n}$.

Proof. Let $\Delta_{n}=\Delta\left(p_{1}, \ldots, p_{n}\right)$ be the minimum $n$-hedron. By the proof of Theorem 1, $p_{n}$ is on the boundary of the convex polyhedron $C$ as well as on 
the surface $\partial(S)$ which is a boundary of the strictly convex set $S$ by Theorem 2 and $S$ is contained in $C$. Take a small ball $B$ around $p_{n}$ with the property that $B \cap \partial(C)$ is contained in a single face of $C$. If $B$ contains a point $\mathbf{v} \in$ $\partial(C) \cap \partial(S)$ other than $p_{n}$, then the segment $\left[\mathbf{v}, p_{n}\right]$ is in $\partial(C) \cap S$ by convexity. However since $S$ is strictly convex, $\left(\mathbf{v}+p_{n}\right) / 2 \in \operatorname{Inn}(S)$ holds, which contradicts $S \subset C$.

\section{Possible shapes of minimum $n$-hedron for $n \leq 12$}

By numerical experiments and Gröbner bases computation, one can give a list of possible shapes of minimum $n$-hedron for $n \leq 12$.

Lemma 5. Let $X$ be a tetrahedron of vertices $K, L, M, N$ and $g$ be the orthogonal projection to the plane $P$ containing $L, M, N$. Let $K$ move in the plane parallel to $P$, keeping its volume $V_{3}(X)$ invariant. Among such $K$, the minimum surface area $A_{3}(X)$ is attained when $g(P)$ is the incenter of the triangle $L M N$.

Proof. Let $h_{1}, h_{2}, h_{3}$ be the signed height ${ }^{4}$ of the point $g(K)$ from the edge $M N, N L, L M$ respectively in the plane $P$ and $h$ is the length of the segment $[K, g(K)]$. Denote by $e_{1}, e_{2}, e_{3}$ the length of the edge $M N, N L$, $L M$ respectively. Then we have $V_{2}(L, M, N)=\left(e_{1} h_{1}+e_{2} h_{2}+e_{3} h_{3}\right) / 2$ and if $V_{2}(L, M, N)$ is fixed, $\left(h_{1}, h_{2}, h_{3}\right)$ gives a coordinate system of points in $P$ under this constraint, i.e., two of $\left\{h_{1}, h_{2}, h_{3}\right\}$ determine the remainder through this relation. Our problem is to minimize

$$
A_{3}(K, L, M, N)=V_{2}(L, M, N)+\frac{1}{2}\left(e_{1} \sqrt{h_{1}^{2}+h^{2}}+e_{2} \sqrt{h_{2}^{2}+h^{2}}+e_{3} \sqrt{h_{3}^{2}+h^{2}}\right)
$$

under $V_{2}(L, M, N)=\left(e_{1} h_{1}+e_{2} h_{2}+e_{3} h_{3}\right) / 2$. Since $\left|h_{i}\right| \rightarrow \infty$ for some $i$ implies $A_{3}(K, L, M, N) \rightarrow \infty$, we may assume that $\left(h_{1}, h_{2}, h_{3}\right)$ are in a compact set of $\mathbb{R}^{3}$. Therefore the minimum of $A_{3}(K, L, M, N)$ exists. Using Lagrange multiplier, we see that the minimum is attained when

$$
\frac{\partial}{\partial h_{i}}\left(A_{3}(K, L, M, N)-\lambda\left(V_{2}(L, M, N)-\left(e_{1} h_{1}+e_{2} h_{2}+e_{3} h_{3}\right) / 2\right)\right)=0,
$$

for $i=1,2,3$. This implies $h_{1} / \sqrt{h_{1}^{2}+h^{2}}=h_{2} / \sqrt{h_{2}^{2}+h^{2}}=h_{3} / \sqrt{h_{3}^{2}+h^{2}}$ and consequently $h_{1}=h_{2}=h_{3}$. Therefore the minimum is attained when $g(P)$ is the incenter of the triangle $L M N$.

\footnotetext{
${ }^{4}$ It is positive in direction to the interior of the triangle $\Delta(L, M, N)$.
} 
This may be a well-known result. One of the referees informed me of a similar discussion in an encyclopedia on elementary geometry, "Kikagaku Dai Jiten" vol. 5, page 440, ed. Shikou Iwata, in Japanese.

Similarly if a pyramid $X$ whose base $k$-gon $B$ is circumscribed about a circle and its apex $K$ moves in the plane parallel to $B$, then $A_{3}(X)$ is minimized when the orthogonal projection of the apex to $B$ is the center of the circle. In fact, let us define $e_{i}, h_{i}$ in a similar manner. Though lengths $h_{i}$ are determined by two parameters, say $h_{1}$ and $h_{2}$, we minimize the surface area function in a less constrained domain

$$
\left\{0 \leq h_{i} \leq C \mid \sum e_{i} h_{i}=2 A_{3}(B)\right\} \subset \mathbb{R}^{k}
$$

with a sufficiently large $C>0$. Then the condition $h_{1}=h_{2}=\cdots=h_{k}$ is attained at the center of the circle under the assumption.

Lemma 6. Any $d+2$ points in $\mathbb{R}^{d}$ is partitioned into two non-empty disjoint sets $U$ and $V$ such that $\Delta(U) \cap \Delta(V) \neq \varnothing$.

Proof. This is due to Radon ([12, Theorem 1.1.5] or [8]). It is an easy consequence of the linear dependence of $v_{i}-v_{d+2}$ for $i=1, \ldots, d+1$ for any point set $\left\{v_{1}, v_{2}, \ldots, v_{d+2}\right\}$.

A $k$ bi-pyramid is a polygon composed of two pyramids sharing the same $k$-gon base joined base to base. A regular $k$ bi-pyramid is a bi-pyramid composed of two congruent regular pyramids sharing the regular $k$-gon base. Its main diagonal is the segment joining two apexes vertically passing the center of the base.

Lemma 7. Among $k$ bi-pyramids 4 whose convex bases are circumscribed about a circle of radius $h$, the minimum

$$
\frac{A_{3}(\Delta)}{V_{3}(\Delta)^{2 / 3}}=\left(3^{7 / 2} k \cot \left(\frac{(k-2) \pi}{2 k}\right)\right)^{1 / 3}
$$

is attained when it is a regular bi-pyramid whose main diagonal has length $\sqrt{8} h$.

Proof. The minimization problem is divided into two pyramids, say, an upper pyramid and a lower pyramid. Let $B$ be the common base polygon. Letting $\theta_{i}(i=1, \ldots, k)$ be the vertex angles of $B$, we obtain $A_{2}(B)=h r$ with $r=2 \sum_{i=1}^{k} \cot \left(\theta_{i} / 2\right)$ and $V_{2}(B)=h A_{2}(B) / 2$. Let $H_{1}$ be the height of the apex of the upper pyramid $\Delta^{\prime}$ to the base $B$, and $H_{2}$ is the one for the lower pyramid $\Delta^{\prime \prime}$. Then we have $V_{3}\left(\Delta^{\prime}\right)=V_{2}(B) H_{1} / 3=h^{2} r H_{1} / 6$. By the discussion after Lemma $5, A_{3}\left(\Delta^{\prime}\right)$ is minimized when the orthogonal projection of the apex to $B$ 
is the center of the circle and

$$
A_{3}\left(\Delta^{\prime}\right)-V_{2}(B)=\frac{1}{2} A_{2}(B) \sqrt{h^{2}+H_{1}^{2}}=\frac{1}{2} h r \sqrt{h^{2}+H_{1}^{2}} .
$$

Let us fix $r$ and minimize $A_{3}(\Delta)$ by selecting $H_{1}, H_{2}$ and $h$ keeping $V_{3}(\Delta)$ invariant. Fixing $h^{2}\left(H_{1}+H_{2}\right) / 6$, the minimum of $h\left(\sqrt{h^{2}+H_{1}^{2}}+\sqrt{h^{2}+H_{2}^{2}}\right) /$ 2 is attained when $H_{1}=H_{2}=\sqrt{2} h$. Now we have $A_{3}(\Delta)=\sqrt{3} h^{2} r$ and $V_{3}(\Delta)=\sqrt{2} h^{3} r / 3$. Thus

$$
\frac{A_{3}(\Delta)}{V_{3}(\Delta)^{2 / 3}}=3^{7 / 6} 2^{-1 / 3} r^{1 / 3} .
$$

Since $\cot (x / 2)$ is convex for $x \in(0, \pi)$, by Jensen's inequality, the minimum of $r$ is achieved by the regular $k$-gon when $\theta_{1}=\theta_{2}=\cdots=\theta_{k}=\pi-2 \pi / k$ and $r=2 k \cot \left(\frac{(k-2) \pi}{2 k}\right)$.

THEOREM 3. We have

$$
\begin{aligned}
& \alpha_{4}=6 \cdot 3^{1 / 6} \approx 7.20562, \\
& \alpha_{5}=3^{5 / 3} \approx 6.24025, \\
& \alpha_{6} \leq \eta_{6}=3^{7 / 6} 2^{2 / 3} \approx 5.71911, \\
& \alpha_{7} \leq \eta_{7}=3^{7 / 6} 5^{5 / 12}(\sqrt{5}-2)^{1 / 6} \approx 5.53841, \\
& \alpha_{8} \leq \eta_{8} \approx 5.42118, \\
& \alpha_{9} \leq \eta_{9} \approx 5.31637, \\
& \alpha_{10} \leq \eta_{10} \approx 5.2533, \\
& \alpha_{11} \leq 5.20713 \\
& \alpha_{12} \leq \eta_{12}=3^{7 / 6}(70-30 \sqrt{5})^{1 / 3} \approx 5.14835,
\end{aligned}
$$

where $\eta_{8}, \eta_{9}, \eta_{10}$ are algebraic numbers of degree 72, 78, 36 respectively. The value $\alpha_{4}$ is attained by a regular tetrahedron and $\alpha_{5}$ by a regular 3 bipyramid.

Our experiments suggest that all the inequalities are equalities, though we did not identify the exact value for $\alpha_{11}$. Several specialists working on computer science told me that brute force optimization does not seem feasible as it has too much free variables for now.

\footnotetext{
${ }^{5}$ For the case $n=6$, see $\S 5(2)$.
} 
Proof. The case $n=4$ may belong to a folklore. At least a written proof is found in Hadwiger [9, p. 273, (187)] using the Steiner symmetrization. Here we give a direct proof. Let $K L M N$ be the minimum 4-hedron. By Lemma 5, projections of $K, L, M, N$ to the corresponding basis triangle must be their incenters respectively. Let $K H$ be the perpendicular from $K$ to $\Delta(L, M, N)$, and $H I, H J$ be the perpendiculars from $H$ to $L M$ and $L N$ respectively. Since $H$ is the incenter of $\Delta(L, M, N)$, we have $H I=H J$, $K I \perp L M, K J \perp L N$. From $K I=K J$ and $I L=J L$, we see that $\angle K L M=\angle K L N$. By cyclic discussion, we see $\angle K L M=\angle K L N=\angle M L N=: \angle L$. Similarly, we see three angles at each vertex of $\Delta(K, L, M, N)$ are identical for all vertices, which are denoted by $\angle K, \angle L, \angle M, \angle N$. Since the sum of angles of triangular faces are all equal to $2 \pi$, we deduce that $\angle K=\angle L=\angle M=\angle N$. Therefore all the faces are regular triangles. This proves the case of the minimum 4-hedron.

For minimum 5-hedron, in light of Lemma 2 we may assume that none of vertices is contained in the convex hull of remaining four vertices. Therefore by Lemma 6, five vertices are divided into two sets $\{K, L, M\}$ and $\{N, O\}$ which satisfy $\Delta(K, L, M) \cap \Delta(N, O) \neq \varnothing$. The problem is therefore reduced to Lemma 7 for $k=3$. This case was also shown in [2, Theorem $5.5]$.

For $n \geq 6$, we performed a random search of the minimum. A rough sketch of the empirical method is

(1) Choose random $n$ points in $\mathbb{R}^{3}$ and determine the combinatorial structure of the convex hull, in particular the valency vector, that is, the multi-set of valencies of vertices.

(2) Iterate process 1 , until we find a valency vector of small variance. Experimentally, we know that $A_{3}(\Delta) / V_{3}(\Delta)^{2 / 3}$ cannot be small if this variance is large.

(3) Select a vertex, an edge or a face of $\Delta$ and minimize $A_{3}(\Delta) / V_{3}(\Delta)^{2 / 3}$ by moving its extremities, keeping the valency vector invariant. If the valency vector changes, then we skip this minimization.

(4) Find two points $v_{1}, v_{2}$ which give the diameter of $\Delta$, and apply an affine transformation to make the diameter a little smaller but keeping the plane orthogonal to $v_{1}-v_{2}$ invariant.

(5) Repeat several times these processes 2, 3 and 4 at random.

Until $n \leq 12$ it seems the above iteration leads us to a possible minimum for a fixed valency vector. Trying many valency vectors, we can guess the target shape. Then we perform algebraic computation to obtain the exact minimum configuration. Taking into account the expected symmetry of the target shape, we set up a system of algebraic equations with a small number of variables. Then we eliminate variables by using some program equipped 
with Gröbner basis computation. We used Mathematica, PARI-GP, and RisaAsir appealing to each advantage. Gröbner basis computation has a lot of subtleties. Successful computation depends heavily on the number of variables, their imposed order, and degree of polynomials. Hereafter we describe our computation but skipping such technical details, giving necessary information to reconfirm the computation.

By our experiments, the target shapes for $\eta_{6}$ and $\eta_{7}$ are attained by regular bi-pyramids as in Lemma 7. The most difficult and interesting shape appears when $n=8$, see Figures 3 and 4 . It is combinatorially equivalent to the Siamese dodecahedron, one of the deltahedra.
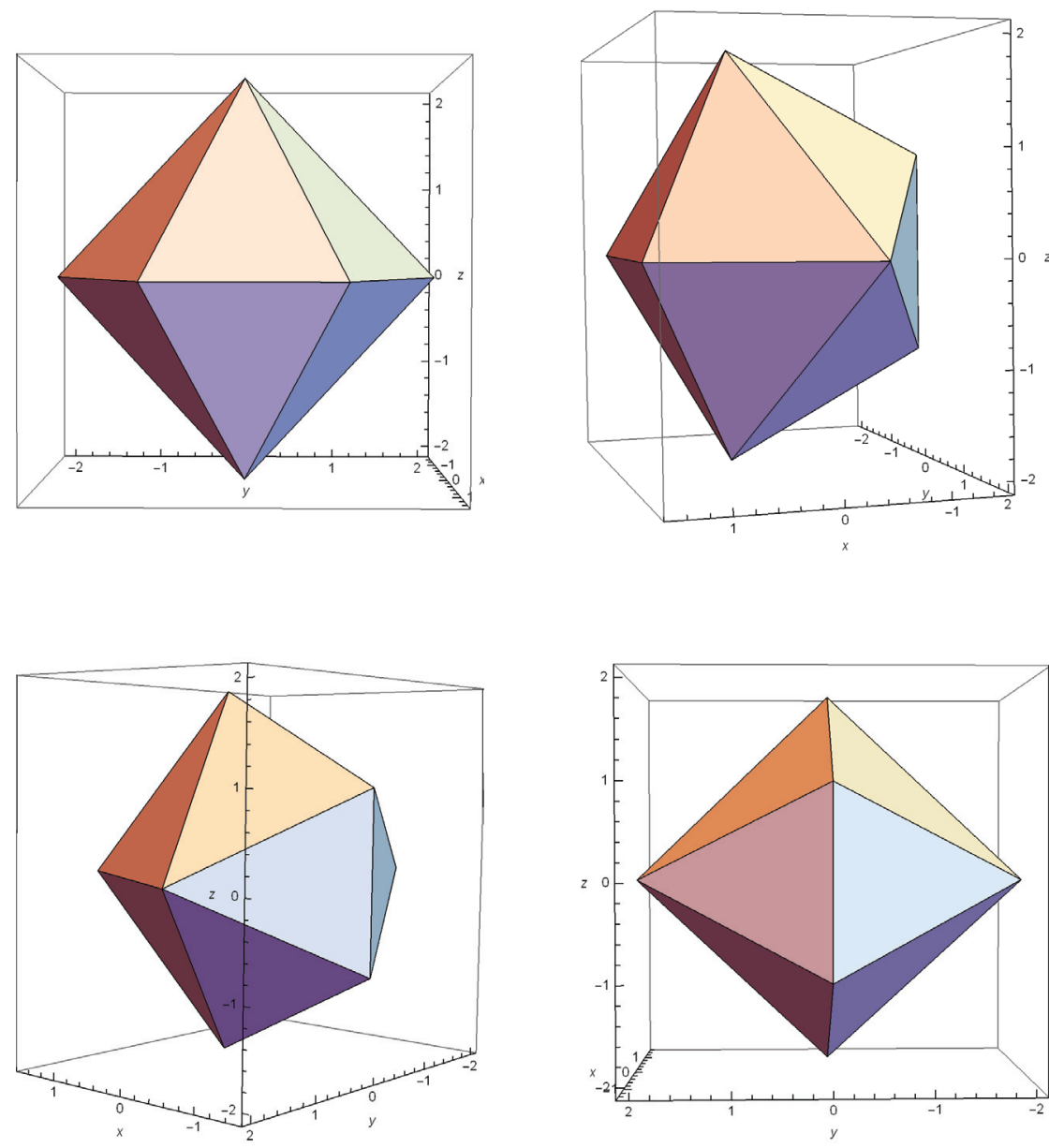

Fig. 3. Minimum 8-hedron 


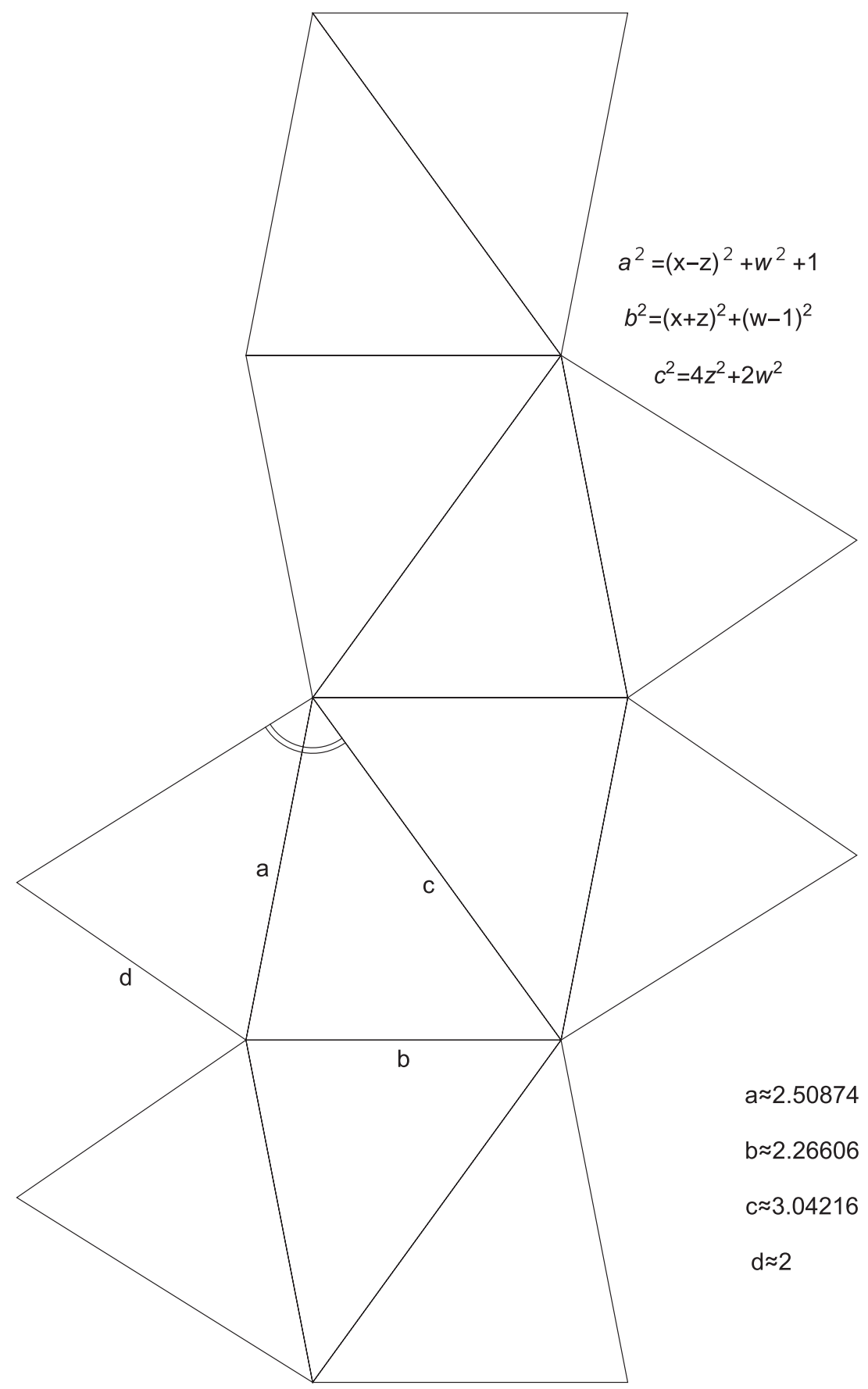

Fig. 4. Origami diagram 
By using numerical minimization, we could guess that 8 points are of the form:

$$
(z, 0, \pm w),(x, \pm 1,0),(-z, \pm w, 0),(-x, 0, \pm 1)
$$

with

$$
w \approx 2.0428, \quad x \approx 1.53525, \quad z \approx 0.476614
$$

Let us consider $w, x, z$ as variables and obtain their exact algebraic representations. We have

$$
A_{3}(\Delta)=4 \sqrt{w^{2}+(x-z)^{2}}+4 \sqrt{(w-1)^{2} w^{2}+w^{2}(x+z)^{2}+(w(x-z)+2 z)^{2}}
$$

and

$$
V_{3}(\Delta)=4 w(x+w x+z) / 3 .
$$

We view $A_{3} / V_{3}^{2 / 3}$ as the function of three variables. Basically our task is to eliminate valuables from

$$
\partial_{x}\left(\frac{A_{3}^{3}}{V_{3}^{2}}\right)=\partial_{w}\left(\frac{A_{3}^{3}}{V_{3}^{2}}\right)=\partial_{z}\left(\frac{A_{3}^{3}}{V_{3}^{2}}\right)=0 .
$$

First transfer problems into the one on polynomials with integer coefficients, by putting

$$
u=\sqrt{w^{2}+(x-z)^{2}}, \quad v=\sqrt{(w-1)^{2} w^{2}+w^{2}(x+z)^{2}+(w(x-z)+2 z)^{2}} .
$$

Then eliminate $u, v$ to find an ideal over $x, w, z$ and perform primary ideal decomposition (this was indispensable for this computation). We obtain the minimal polynomials ${ }^{6}$ of $w, x^{2}, z^{2}$ :

$$
\begin{gathered}
8-40 t-32 t^{2}+268 t^{3}-14 t^{4}+378 t^{5}-916 t^{6}+874 t^{7} \\
-265 t^{8}-314 t^{9}+374 t^{10}-150 t^{11}+21 t^{12} \\
-1500625+246891400 t-6498924184 t^{2}+197676252320 t^{3}-549916476544 t^{4} \\
+9593743607488 t^{5}-37068998078592 t^{6}+43451585720832 t^{7} \\
+6412940883200 t^{8}-47369088623616 t^{9}+34505601388544 t^{10} \\
-10887830962176 t^{11}+1413638553600 t^{12},
\end{gathered}
$$

\footnotetext{
${ }^{6}$ Minimal polynomials of $x$ and $z$ are the ones subject to substitution $t \rightarrow t^{2}$.
} 


$$
\begin{aligned}
& -881721+14088624 t-507815656 t^{2}+22228266304 t^{3}-345876361600 t^{4} \\
& +2163078191936 t^{5}-5229062814592 t^{6}+2885777661952 t^{7}+604100406528 t^{8} \\
& +284044459008 t^{9}-1111813844992 t^{10}+65086242816 t^{11}+157070950400 t^{12}
\end{aligned}
$$

The minimal polynomial of $A_{3}(\Delta)^{6} /\left(V_{3}(\Delta)\right)^{4}$ is

$$
\begin{aligned}
& 846253032058341803633618097683156083357246027504784634537836544 \\
- & 145765911302088136407360046924472940590350227969907327078760448 t \\
+ & 44739094836549297939345827315732094525400511681413644681216 t^{2} \\
- & 5444218664651134627342263572192722894788633799480098816 t^{3} \\
+ & 381929202246269536064619254896305729053865712762224 t^{4} \\
- & 23215968331655851588483378342178431615039134384 t^{5} \\
+ & 908544689594387775769635417411363042641304 t^{6} \\
- & 26376155703404842068063899980163109720 t^{7} \\
+ & 639590587552165626186327476412759 t^{8} \\
- & 9114814042610279966292752064 t^{9}+144758783681628174471168 t^{10} \\
- & 130494391161126912 t^{11}+4980736000 t^{12} .
\end{aligned}
$$

A non-trivial coincidence of two angles indicated in Figure 4 is confirmed by algebraic computation of cosine values of the angles. One can also confirm numerically that this minimum shape is rigid, see Section 5.

For $\eta_{9}$, consider a regular triangular prism and put three identical 4-pyramids to each of rectangular side faces whose centroid is the foot of the perpendicular from the apex of the pyramid, see Figure 5 (a). Let the edge length of the regular triangle be 1 . Then the height of the prism $b$, and the height of the 4-pyramid $h$ are expected to be

$$
b \approx 1.04725, \quad h \approx 0.413823 .
$$

We have

$$
A_{3}(\Delta)=\frac{\sqrt{3}}{2}+3 b \sqrt{h^{2}+\frac{1}{4}}+3 \sqrt{h^{2}+\frac{b^{2}}{4}}
$$

and

$$
V_{3}(\Delta)=\frac{\sqrt{3} b}{4}+b h .
$$


We treat $A_{3}(\Delta) / V_{3}(\Delta)^{2 / 3}$ as a function of two variables $b$ and $h$, and apply the elimination of variables as we did in $n=8$. Note that in order to treat $\sqrt{3}$, we also introduce another variable $s$ and the polynomial $s^{2}-3$ to be added in the ideal. The minimal polynomials of $b^{2}$ and $h^{2}$ are

$$
\begin{aligned}
& -3600-9384 t+157415 t^{2}+1871849 t^{3}-3005515 t^{4}-3048555 t^{5} \\
& +7100157 t^{6}-716904 t^{7}-5370867 t^{8}+3887865 t^{9}-810945 t^{10} \\
& -53622 t^{11}+17415 t^{12}+2187 t^{13}
\end{aligned}
$$

and

$$
\begin{aligned}
& -27-216 t-5688 t^{2}+99268 t^{3}+2629424 t^{4}-11859776 t^{5}-198587904 t^{6} \\
& +641098752 t^{7}+2269974528 t^{8}+3790651392 t^{9}-43985534976 t^{10} \\
& +74140876800 t^{11}-37371248640 t^{12}+5435817984 t^{13}
\end{aligned}
$$

respectively. The minimal polynomial of $A_{3}^{6} / V_{3}^{4}$ is

$$
\begin{aligned}
& -8741200275671730192755167246352564248392781977833773782269952 \\
& +5692272790315788765597663433429575175625193067065671949484032 t \\
& +1663401637275489431763071207791450034909825698981382756499456 t^{2} \\
& +205636897183575223972130099822721877708248944269405343514624 t^{3} \\
& -29496333327693613396843751515776856015704029599701614592 t^{4} \\
& -58714195329202332973530206007453465620049796957569024 t^{5} \\
& -13596161545396297014562622838466932898374596846592 t^{6} \\
& -847586880386300377059351613641377507384112384 t^{7} \\
& -58377287904203791631778906263194550638656 t^{8} \\
& +3993703760487214498878732921512576256 t^{9} \\
& -12610065164386918027558684269276 t^{10} \\
& +4281392126518694452576397473 t^{11}-20704119330241635606528 t^{12} \\
& +21761395104153600 t^{13} .
\end{aligned}
$$

For $\eta_{10}$, prepare an anti-prism, a convex hull of a square and its parallel square rotated by $\pi / 4$, and put two identical regular 4-pyramids on the two 


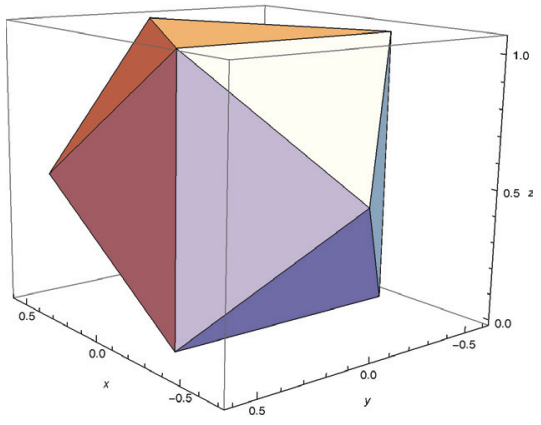

(a) $n=9$

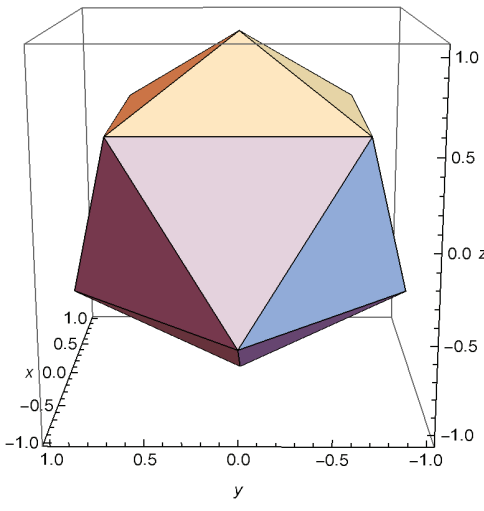

(b) $n=10$

Fig. 5. Minimum polyhedron

parallel squares, see Figure 5 (b). We introduce a coordinate of 10 points:

$$
( \pm 1,0,-h),(0, \pm 1,-h),\left( \pm \frac{1}{\sqrt{2}}, \pm \frac{1}{\sqrt{2}}, h\right),(0,0, \pm z)
$$

with

$$
h \approx 0.541397, \quad z \approx 1.02619
$$

and minimize

$$
\frac{A_{3}^{3}}{V_{3}^{2}}=\frac{36\left(\sqrt{3-2 \sqrt{2}+8 h^{2}}+\sqrt{1+2 h^{2}-4 h z+2 z^{2}}\right)^{3}}{(h+\sqrt{2} h+z)^{2}} .
$$

The minimal polynomials of $h^{2}, z^{2}, A_{3}^{6} / V_{3}^{4}$ are

$$
\begin{gathered}
1+48 t+144 t^{2}-16128 t^{3}-31296 t^{4}+273408 t^{5}+28672 t^{6}, \\
47089+1130960 t-1729392 t^{2}+2846464 t^{3}-1889856 t^{4}-277504 t^{5}+28672 t^{6},
\end{gathered}
$$

and

- $9592639401335565227088041861971968+362253880325110957404812476416 t$

$-4924615865029090098020352 t^{2}+462296427139672731648 t^{3}$

$-713296009601244 t^{4}+274678452 t^{5}+t^{6}$,

respectively. We also obtained the conjectural shape for $n=11$ by experiments. It is a convex hull of

$$
\left(x_{1}, 0, \pm 1\right),\left(x_{2}, \pm y_{1}, 0\right),\left(-x_{3}, \pm y_{2}, 0\right),\left(-x_{4}, \pm y_{3}, \pm z\right),\left(-x_{5}, 0,0\right)
$$


with

$x_{1} \approx 1.15135, \quad x_{2} \approx 0.617047, \quad x_{3} \approx 0.91681, \quad x_{4} \approx 0.550702, \quad x_{5} \approx 1.98113$,

$$
y_{1} \approx 1.4264, \quad y_{2} \approx 1.34059, \quad y_{3} \approx 0.845054, \quad z \approx 1.38959
$$

and

$$
A_{3} / V_{3}^{2 / 3} \approx 5.207134373504469,
$$

see Figures 6 and 7. It has 18 faces, which follows from Euler's formula and the fact that all faces are triangles. The shape is combinatorially equivalent to a polyhedron obtained by merging two adjacent vertices of the regular icosahedron into one.

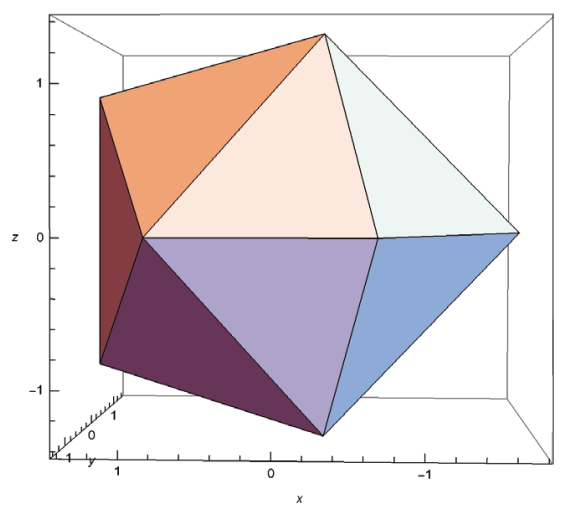

Fig. 6. $n=11$ : From $y$-axis direction

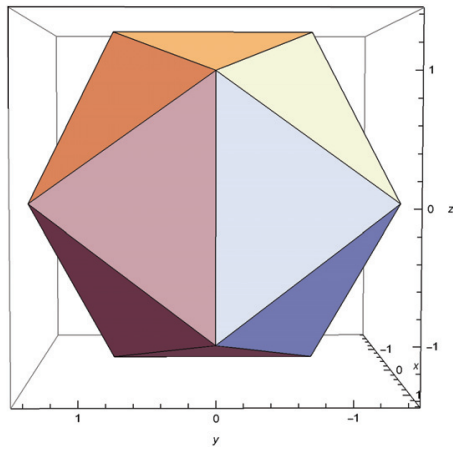

(a) From $x$-axis positive direction

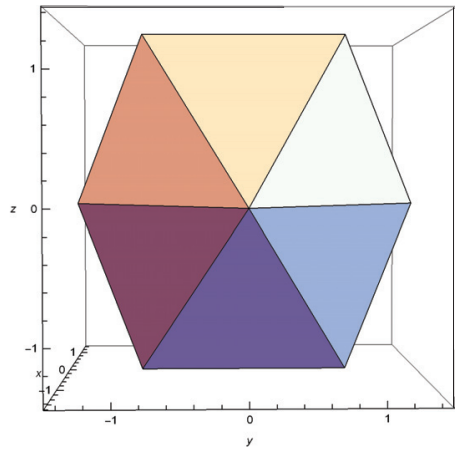

(b) From $x$-axis negative direction

Fig. 7. Minimum 11-hedron 
We could not make the coordinates algebraic, because the expected symmetry group $(\mathbb{Z} / 2 \mathbb{Z})^{2}$ is too small, and the number of valuables is too large.

The minimum 12-hedron is of course expected to be the regular icosahedron with

$$
A_{3} / V_{3}^{2 / 3}=3^{7 / 6}(70-30 \sqrt{5})^{1 / 3} \approx 5.14835
$$

\section{Problems}

We give a list of intriguing problems.

(1) Can we give an asymptotic estimate for the convergence of $\left(\alpha_{n}\right)$ ?

(2) Prove our candidates minimum for $i=6,7,8,9,10,12$. (Added in Revision: the validity for $i=6$ is confirmed in [3]).

(3) Is the minimum $n$-hedron $\Delta\left(p_{1}, \ldots, p_{n}\right)$ rigid? We say that $\Delta\left(p_{1}, \ldots\right.$, $\left.p_{n}\right)$ is rigid if it does not allow deformation of $n-3$ vertices, i.e., there exists a positive $r$ so that for any subset $I$ of $\{1, \ldots, n\}$ of cardinality $n-3$, if $\Delta\left(x_{1}, \ldots, x_{n}\right)$ is a minimum $n$-hedron with $\left\|x_{i}-p_{i}\right\|<r$ for $i \in I$ and $x_{i}=p_{i}$ for $i \notin I$, then $x_{i}=p_{i}$ holds for all $i$.

(4) Is the symmetry group of the minimum $n$-hedron non-trivial for all $n$ ? Can it have a chirality, i.e., can its symmetry group in $O(3)$ and that in $S O(3)$ be different?

(5) Is $\partial(S)$ in Theorem 2 defined by strongly convex functions?

\section{Acknowledgement}

The author wishes to express his deepest gratitude to anonymous referees for pointing out mistakes, missing references and very careful reading of the original manuscript.

\section{References}

[1] H. H. Bauschke, Y. Lucet, and H. M. Phan, On the convexity of piecewise-defined functions, ESAIM Control Optim. Calc. Var. 22 (2016), no. 3, 728-742.

[2] K. Böröczky and K. J. Böröczky, Isoperimetric problems for polytopes with a given number of vertices, Mathematika, 43 (1996), no. 2, 237-254.

[3] K. J. Böröczky and A. Kovács, The isoperimetric problem for 3-polytopes with six vertices, arXiv:1901.02160.

[4] L. Fejes Tóth, Über zwei Maximumaufgaben bei Polyedern, Tôhoku Math. Journal (1-st series) 46 (1939), 79-83, Published under the name: Ladislaus Fejes.

[5] L. Fejes Tóth, Über einige extremaleigenschaften der regularen Polyeder und des gleichseitigen Dreiecksgitters, Annal. Scuola Normal. Super. Pisa, Cl. Sci. 13 (1948), 51-58, Published under the name: László Fejes.

[6] L. Fejes Tóth, The isepiphan problem for $n$-hedra, Amer. J. Math. 70 (1948), 174-180. 
[7] L. Fejes Tóth, Lagerungen in der Ebene auf der Kugel und im Raum Zweite verbesserte und erweiterte Auflage, Die Grundlehren der mathematischen Wissenschaften, Band 65. SpringerVerlag, Berlin-New York, 1972, xi+238 pp. (Japanese translation "Haichi no Mondai" is published by Misuzu Shobou. See p. 141)

[8] B. Grünbaum, Convex polytopes, Second ed., Graduate Texts in Mathematics, vol. 221, Springer-Verlag, New York, 2003, xvi+468.

[9] H. Hadwiger, Vorlesungen über Inhalt, Oberfläche und Isoperimetrie, Springer-Verlag, Berlin-Göttingen-Heidelberg 1957, xiii+312 pp.

[10] L. Lindelöf, Propriétés générales des polyèdres qui, sous une étendue superficielle donnée, renferment le plus grand volume, St. Petersburg Bull. Ac. Sc. 14 (1869), 257-269.

[11] H. Minkowski, Allgemeine lehrsätze über die konvexen polyeder, Nachr. Ges. Wiss. Göttingen (1897), 198-219. Gesammelte Abhandlungen, vol. 2, Chelsea, New York, 1967, pp. $103-121$.

[12] R. Schneider, Convex bodies: the Brunn-Minkowski theory, Encyclopedia of Mathematics and its Applications, vol. 44, Cambridge University Press, Cambridge, 1993.

\author{
Shigeki Akiyama \\ Institute of Mathematics \\ University of Tsukuba
}

1-1-1 Tennodai, Tsukuba, Ibaraki, 305-8571 Japan

E-mail: akiyama@math.tsukuba.ac.jp

URL: http://math.tsukuba.ac.jp/ akiyama/ 